\title{
Low-Order Controller Design for Haptic Systems under Delayed Feedback
}

\author{
Bogdan Liacu ${ }^{*, * * *}$ Ahmet Taha Koru ${ }^{* *}$ Hitay Ozbay ${ }^{* *}$ \\ Silviu-Iulian Niculescu * Claude Andriot ${ }^{* * *}$ \\ * Laboratoire des Signaux et Systèmes (LSS), CNRS-SUPELEC, 3 rue Joliot \\ Curie, 91192 Gif-sur-Yvette France(e-mail: bogdan.liacu@supelec.fr, \\ Silviu.Niculescu@lss.supelec.fr) \\ ** Dept. of Electrical \& Electronics Eng, Bilkent University, Ankara, Turkey \\ (e-mail: koru@ee.bilkent.edu.tr,hitay@ee.bilkent.edu.tr) \\ *** CEA, LIST, Interactive Robotics Laboratory, Fontenay aux Roses, \\ F-92265,France (e-mail: claude.andriot@cea.fr)
}

\begin{abstract}
In this paper, we consider PD controller design for haptic systems under delayed feedback. More precisely, we present a complete stability analysis of a haptic system where local dynamics are described by some second-order mechanical dynamics. Next, using two optimization techniques $(H$ and stability margin optimization) we propose an optimal choice for the controller gains. The derived results are tested on a three degree of freedom real-time experimental platform to illustrate the theoretical results.
\end{abstract}

Keywords: Teleoperation, time delay, H-infinity optimization, stability limits, PID control.

\section{INTRODUCTION}

During the last decade, virtual environments have become very popular and are largely used in many domains as, for example, prototyping (see, for instance, Figure 1.a for an appropriate example of prototyping using haptic interfaces and virtual environment Lecuyer et al. (2003)), training for different devices and assistance in completing difficult tasks (see Figure 1.b for some virtual environment used for task assistance/supervision David et al. (2007), Gosselin et al. (2010)).

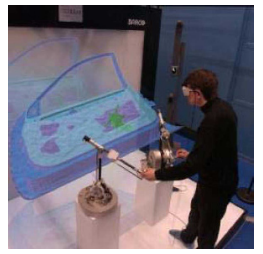

a. Virtual Prototyping.

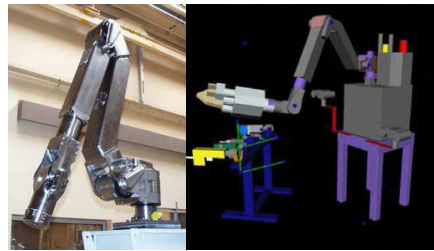

b. Virtual Assistance/Supervision.
Fig. 1. Examples of Virtual Environments Applications

Understanding the interaction between humans and robots is at the origin of developing several control schemes for teleoperation systems. Roughly speaking, teleoperation extends, at some level, the human capacity in manipulating objects remotely by providing the corresponding operator with similar conditions as those encountered at the remote location (see, for instance, the surveys Hokayem and Spong (2006), Sheridan (1993)). Among the recent applications, we may cite telesurgery and space telerobotics (see, for instance, Aziminejad et al. (2008) and the references therein), both involving long distance communication between master and slave devices. Furthermore, in both cases, haptic feedback proved its potential in improving corresponding task performance. In this context, delays appear as natural components of the closed-loop schemes in order to describe some of the dynamics induced by the communication channels with strong impact on (asymptotic) stability and transparency (i.e. the capability as well as the impression of operating directly on a remote environment independently of the presence of master and slave units Lawrence (1993), Yokokohji and Yoshikawa (1994)). In is worth mentioning that, in haptic systems, excepting the communication channel, delays may appear as intrinsic components of the processing time for the virtual reality environment. More precisely, in free motion, the delay effect can be felt by the viscosity phenomenon (high force feedback felt at the haptic interface end) and such a property is completely lost in the case of a "hard"-contact with the environment.

In the sequel, we will focus on the closed-loop stability analysis of some class of practical bilateral haptic systems coupled with a virtual environment by using a standard proportionalderivative (PD) control law. The delays in the communication channels are assumed to be constant and, as we will see in the sequel, only the overall delay (the sum of the forward and backward delays) needs to be known. There exists an abundant literature on PID control for time-delay systems (see for instance, O’Dwyer (2000), Silva et al. (2005) and the references therein). If the stability analysis in closed-loop makes use of classical tools, the approach we are proposing in this paper for guaranteeing performance is original. More precisely, by exploiting the particular structure of the closed-loop quasipolynomials, we focus on the computation of the the optimal controller gains by using two particular frequency-domain techniques: $H$-based design and fragility analysis. Here, by fragility, we simply understand the deterioration of closed-loop stability due to small variations of the system parameters (see, for instance, Alfaro (2007), Keel and Bhattacharyya (1997), and Makila et al. (1998) for further details on such topics). Finally, the derived control law are validated on some illustrative example involving a virtual spherical mass moving in an appropriate 3D virtual scene and the study is performed by 
considering a complete scenario from free to some restricted motions.

The remaining paper is organized as follows: in Section 2, we present a general haptic system scheme including communication channels. Next, section 3 is devoted to the stability analysis in closed-loop in the presence of PD control laws. Section 4 focuses on an appropriate optimal choice for the controller parameters by using the (frequency-domain) approaches mentioned above. The experimental validation of the proposed methodology is discussed in section 5 on a simple three degree of freedom haptic system. Finally, some concluding remarks end the paper.

\section{SYSTEM DESCRIPTION}

In figure 2 we present the general scheme of a haptic system. The ideal haptic system should satisfy simultaneously the following conditions:

- first, the position tracking error has to be as small as possible between the haptic interface and the virtual object,

- second, the system has to have a high degree of transparency, i.e. in the "free" motion case, the force feedback felt at the haptic interface end must be as small as possible and in the case of a "hard"-contact, a stiff response is desired.

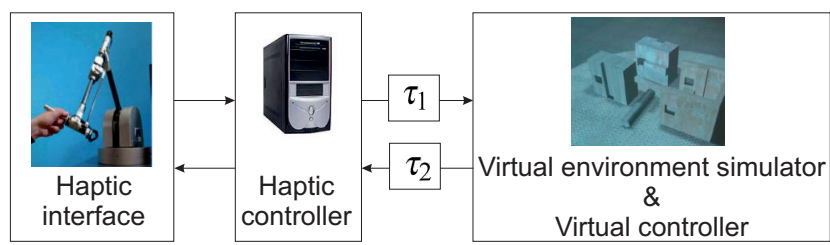

Fig. 2. General Scheme of a Haptic System

Figure 3 presents the general control scheme of a haptic interface and a virtual environment including control feedback.

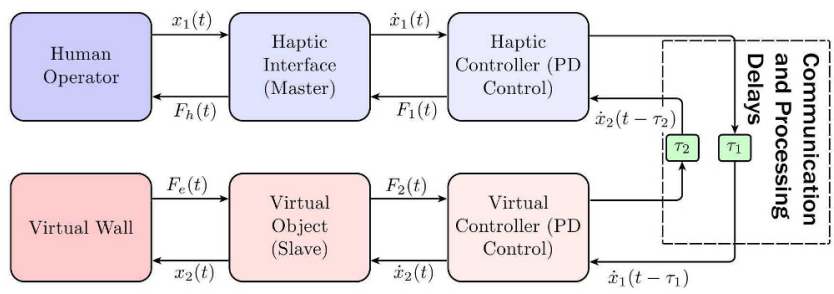

Fig. 3. General PD control scheme for haptic systems.

We will start from the classical dynamic (nonlinear) equations of motion for two similar robots in the haptics framework. More precisely, the corresponding dynamics write as:

$$
\begin{aligned}
& M_{1}\left(x_{1}\right) \ddot{x}_{1}(t)+C_{1}\left(x_{1}, \dot{x}_{1}\right) \dot{x}_{1}=-F_{1}(t)+F_{h}(t), \\
& M_{2}\left(x_{2}\right) \ddot{x}_{2}(t)+C_{2}\left(x_{2}, \dot{x}_{2}\right) \dot{x}_{2}=-F_{2}(t)+F_{e}(t),
\end{aligned}
$$

where $x_{1}, x_{2}$ are the haptic interface/virtual object position, $F_{h}, F_{e}$ are the human/environmental forces, $F_{1}, F_{2}$ are the force control signals, $M_{1}, M_{2}$ are the symmetric and positive-definite inertia matrices, and $C_{1}, C_{2}$ are the Coriolis matrices of the haptic interface and virtual object systems, respectively. The main idea can be resumed to using two similar PD controllers, one for controlling the haptic interface and another for the (corresponding) virtual object. In such a configuration, we have:

$$
\begin{aligned}
& F_{1}(t)=\underbrace{K_{d}\left(\dot{x}_{1}(t)-\dot{x}_{2}(t-2)\right)}_{\text {delayed D-action }} \underbrace{+K_{p}\left(x_{1}(t)-x_{2}(t-2)\right)}_{\text {delayed P-action }}, \\
& F_{2}(t)=\underbrace{K_{d}\left(\dot{x}_{2}(t)-\dot{x}_{1}(t-1)\right)}_{\text {delayed D-action }} \underbrace{+K_{p}\left(x_{2}(t)-x_{1}\left(t-{ }_{1}\right)\right)}_{\text {delayed P-action }},
\end{aligned}
$$

where 1,2 are the forward and backward finite constant delays and $K_{p}, K_{d}$ are the PD control gains.

\section{STABILITY ANALYSIS}

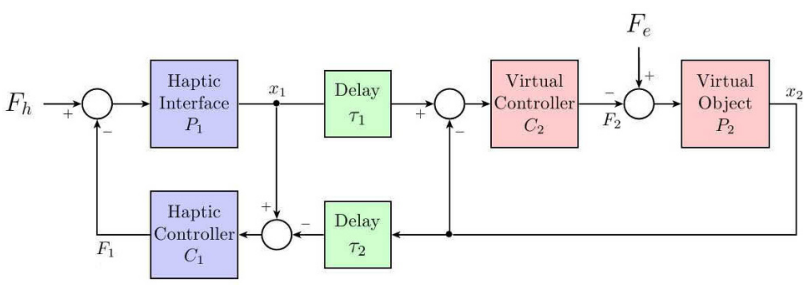

Fig. 4. Bilateral Haptic System.

From Figure 4, the equations describing the system response can be written as follows:

$$
\begin{aligned}
& X_{1}(s)=P_{1}(s)\left(F_{h}(s)-C_{1}(s)\left(X_{1}(s)-e^{-{ }^{2} s} X_{2}(s)\right)\right), \\
& X_{2}(s)=P_{2}(s)\left(-F_{e}(s)+C_{2}(s)\left(-X_{2}(s)+e^{-{ }_{1} s} X_{1}(s)\right)\right),
\end{aligned}
$$

where $X_{i}(s)$ denotes the Laplace transform of the time signal $x_{i}(t), i=1,2$; similarly for $F_{h}(s)$ and $F_{e}(s)$; Here, $1>0$ and $2>0$ denote the corresponding (forward and backward) time delays. Transfer functions $P_{i}(s)$ and $C_{i}(s)$ are taken as follows (position available for measurement and PD structure for the control law):

$$
\begin{gathered}
P_{1}(s)=P_{2}(s)=\frac{1}{s(m s+b)}=: P(s), \\
C_{1}(s)=C_{2}(s)=K_{p}+K_{d} s=: C(s) .
\end{gathered}
$$

As far as the internal stability analysis is concerned, the above system is equivalent to a system where the controller is PI (of the form $K_{d}+K_{p} / s$ ), and the process (measured) variable is represented by the velocity, i.e., process given by: $(m s+b)^{-1}$.

By rearranging (5) and (6) above, we get:

$$
\begin{gathered}
{\left[\begin{array}{cc}
1+P_{1}(s) C_{1}(s) & -P_{1}(s) C_{1}(s) e^{-{ }_{2} s} \\
-P_{2}(s) C_{2}(s) e^{-}{ }_{1} s & 1+P_{2}(s) C_{2}(s)
\end{array}\right]\left[\begin{array}{l}
X_{1}(s) \\
X_{2}(s)
\end{array}\right]} \\
=\left[\begin{array}{c}
P_{1}(s) F_{h}(s) \\
-P_{2}(s) F_{e}(s)
\end{array}\right]
\end{gathered}
$$

Therefore, with the process (plant) and controller definitions (7) and (8), the characteristic equation of the feedback system rewrites as follows:

$$
(1+P(s) C(s))^{2}-(P(s) C(s))^{2} e^{-\left({ }_{1}+{ }_{2}\right) s}=0,
$$

which is simply equivalent to:

$$
1(s) \quad 2(s)=0
$$

where:

$$
\begin{aligned}
& { }_{1}(s):=\left(1+P(s) C(s)+P(s) C(s) e^{-s}\right), \\
& { }_{2}(s)=:\left(1+P(s) C(s)-P(s) C(s) e^{-s}\right),
\end{aligned}
$$

and $:=\frac{(1+2)}{2}$. 
Remark 1. An analysis of equations of the form (11) has been given in Shayer and Campbell (2000). Different approaches for handling such a control problem can be found in Liacu et al. (2010) (closed-loop stability analysis in the controllergains parameter space, see also Saeki (2007)), Michiels and Niculescu (2007) and the references therein (optimal delay bound as a function of parameters). In the sequel we are considering a different approach that makes use of classical tools from control theory (such as gain and phase margins) in order to perform the stability analysis of such a feedback system.

Since $(1+P C)^{-1}$ is a stable transfer function, from (11) it is worth mentioning that the feedback system is stable if and only if the following two equations do not have zeros in $\overline{\mathbb{C}}_{+}$:

$$
\begin{aligned}
& 1+G(s)\left(\frac{1-e^{-s}}{s}\right)=0, \text { where } G(s)=\frac{K_{p}+K_{d} s}{m s+b}, \\
& 1+T(s) e^{-s}=0, \text { where } T(s)=\frac{K_{p}+K_{d} s}{s(m s+b)+K_{p}+K_{d} s} .
\end{aligned}
$$

Now define:

$$
K:=\frac{K_{p}}{b}, \quad{ }_{c}:=\frac{K_{d}}{K_{p}}, \quad p:=\frac{m}{b},
$$

then $G(s)$ and $T(s)$ can be re-written as:

$$
\begin{gathered}
G(s)=K \frac{1+{ }_{c} s}{1+{ }_{p} s}, \\
T(s)=\frac{K\left(1+{ }_{c} s\right)}{\left.p^{s^{2}+(1+c}\right) s+K} .
\end{gathered}
$$

Further, a frequency normalization can be made:

$$
\widehat{s}=p s,
$$

and introduce new definitions:

$L:=\frac{1}{K_{p}}=\frac{b^{2}}{m K_{p}}, \quad:=\frac{c}{p}=\frac{b K_{d}}{m K_{p}}, \quad h:=\frac{-}{p}=\frac{(1+2) b}{2 m}$

so that the characteristic equations (12) and (13) can be rewritten as:

$$
\begin{aligned}
& 1+\frac{1}{L} \frac{(1+\widehat{s})}{(1+\widehat{s})}\left(\frac{1-e^{-h \widehat{s}}}{\widehat{s}}\right)=0, \\
& 1+\frac{(1+\widehat{s})}{\left(L \widehat{s}^{2}+(L+) \widehat{s}+1\right)} e^{-h \widehat{s}}=0 .
\end{aligned}
$$

The next step is to find the controller parameters $L$ and (which define $K_{p}$ and $K_{d}$ ), as functions of $h$, that place all the roots of (18) and (19) in $\mathbb{C}_{-}$. In what follows without any lack of generality only the case where $K_{p}$ and $K_{d}$ are positive, i.e. $L>0$ and $>0$ is considered. It is worth mentioning that, in practice, such a situation occurs most of the cases. As shown in Appendix A, the system is stable independent of delay $h$ if

$\geq 1$. Furthermore, the analysis for the case $<1$ reduces to the following. Define:

$$
\begin{aligned}
& g_{c}(x)=\frac{-2\left(\tan ^{-1}(x)-\tan ^{-1}(x)\right)}{x}, \\
& g_{p}(x)=\frac{2\left(-\left(\tan ^{-1}(x)-\tan ^{-1}(x)\right)\right)}{x} .
\end{aligned}
$$

Clearly, $g_{p}$ and $g_{c}$ are uniformly decreasing functions and $g_{p}(x)>g_{c}(x)$ for all $x>0$. So, if $p$ is defined as the solution of the equation $g_{p}(x)=h$ and $\quad{ }_{o}$ as the solution of the equation $g_{c}(x)=h$, then $o<p$ and hence, for $<1$, the feedback system shown in Figure 4 is stable if and only if ${ }_{c}<{ }_{o}$, which is equivalent to:

$L>\frac{2(1-)}{1+2}, \quad$ where $\quad o>0$ is the solution of $g_{c}(x)=h$.

In conclusion, the following result is obtained:

Theorem 1. The bilateral haptic system is asymptotically stable independent of the delay values $(1,2)$ if and only if the controller gains satisfy the condition:

$$
K_{d} \geq \frac{m}{b} K_{p} .
$$

Furthermore, when $K_{d} / K_{p}<m / b$, the closed-loop system is stable if and only if:

$$
\frac{2}{b^{2}} \frac{m K_{p}-b K_{d}}{1+\underset{0}{2}}<1
$$

where $0>0$ is the solution of the equation:

$$
\frac{-2\left(\tan ^{-1}(x)-\tan ^{-1}\left(\frac{b K_{d}}{m K_{p}} x\right)\right)}{x}=\frac{(1+2) b}{2 m} .
$$

From the conditions of Theorem 1, the allowable range of $m K_{p} / b^{2}$ and $K_{d} / b$ for all $b / m>0$ can be determined. The corresponding stability region is shown for three different time delay values in Figure 5.

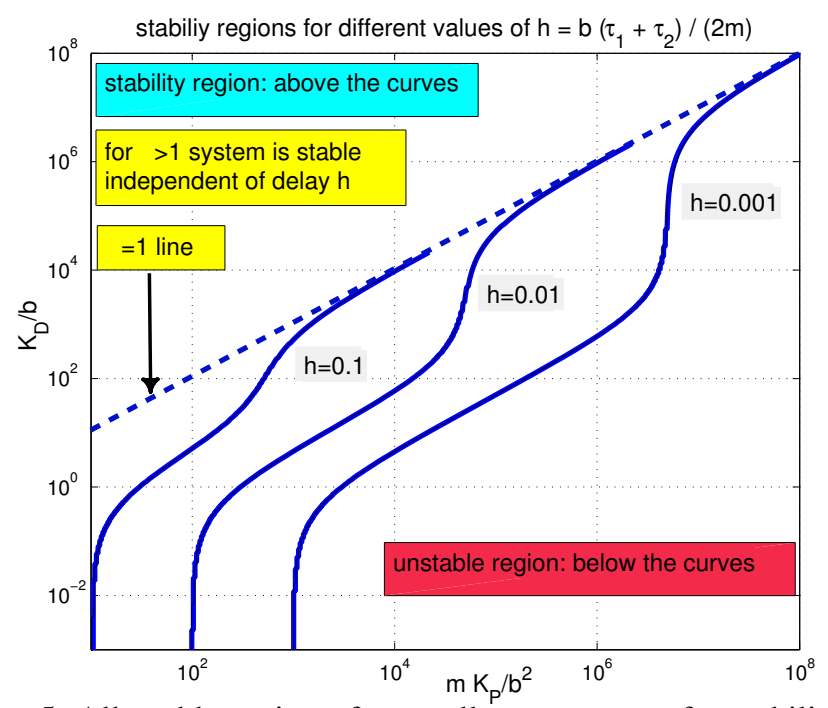

Fig. 5. Allowable region of controller parameters for stability of the bilateral haptic system.

\section{OPTIMAL GAINS}

In this section we discuss optimal gains $K_{p}$ and $K_{d}$ from different perspectives.

\subsection{H -based design}

Let us define the position tracking error:

From (9) we compute:

$$
e(t):=x_{1}(t)-x_{2}(t) \text {. }
$$

$$
E(s)=\frac{P(s)}{1+P(s) C(s)+P(s) C(s) e^{-s}}\left(F_{h}(s)+F_{e}(s)\right) .
$$


While trying to make the error small we may be forced to use high command signals which may lead to actuator saturation. Since large control signals are not desirable, we also want to penalize the control. Again, from (9), the output of the controller, $F_{2}(t)$, on the virtual side can be computed as:

$$
F_{2}(s)=C(s)\left(e^{-s} X_{1}(s)-X_{2}(s)\right) .
$$

In particular, when $F_{e}=0$ we have:

$$
\left[\begin{array}{c}
E(s) \\
F_{2}(s)
\end{array}\right]=\left(\frac{T(s)}{1+T(s) e^{-s}}\right)\left[\begin{array}{c}
1 / C(s) \\
\frac{e^{-s}}{1+P(s) C(s)\left(1-e^{-s}\right)}
\end{array}\right] F_{h}(s),
$$

where $T(s)=P(s) C(s)(1+P(s) C(s))^{-1}$. Therefore, optimal gains from the $H$ control point of view are the ones which solve the problem:

$\min _{K_{p}, K_{d}}\left\|\frac{P(s)}{1+P(s) C(s)\left(1+e^{-s}\right)}\left[\frac{C(s)}{\left(1+P(s) C(s)\left(1-e^{-s}\right)\right)}\right]\right\|$

where is a design parameter which represents the trade-off between small tracking error $e$ and small control action $F_{2}$. Depending on the values of we obtain the optimal $K_{p}$ and $K_{d}$, for each fixed $m=1, b=0.1$ and $=0.05$, as shown in Table 1.

Table 1. $H$ optimal gains for different

\begin{tabular}{||c||c|c|c|c|c|c||}
\hline$b^{2}$ & 0.01 & 0.1 & 1 & 10 & 50 & 100 \\
\hline \hline$K_{p}$ & 0.8 & 17.1 & 85.0 & 246 & 305 & 310 \\
\hline$K_{d}$ & 8.8 & 10.2 & 15.2 & 43 & 55 & 51 \\
\hline
\end{tabular}

We see that for large values of (emphasizing tracking performance, i.e., trying to make $\|e\|_{2}$ small compared to $\left\|F_{2}\right\|_{2}$ ) $H$ optimal gains are in the order of $K_{p} \in[240,310]$ and $K_{d} \in[40,55]$. In the next section we will compare these values with another set of gains obtained from a different optimality criterion.

\subsection{Stability margin optimization}

One of the stability conditions is:

$$
\left(\frac{b^{2}}{m K_{p}}\right)\left(\frac{1+\underset{p}{2}}{2(1-\quad)}\right)>1
$$

Note that $o_{o}<{ }_{p}$ so, if we define:

$$
G M_{1}:=\left(\frac{b^{2}}{m K_{p}}\right)\left(\frac{1+\stackrel{2}{o}}{2(1-\quad)}\right),
$$

then $G M_{1}>1$ implies (28). So, we will try to make $G M_{1}$ as large as possible. On the other hand, for large bandwidth in the system (fast response) we require that ${ }_{c}$ is as large as possible, i.e.:

$$
{ }_{c}^{2}+1=\frac{m K_{p}}{b^{2}} 2(1-\quad),
$$

should be as large as possible. But this conflicts with $G M_{1}$ should be large condition. So, we will blend these two conflicting objectives and try to:

$$
\operatorname{maximize} \min \left\{1\left({ }_{c}^{2}+1\right), \frac{1}{1} G M_{1}\right\},
$$

where 1 assigns a relative weight for each component of the problem. The solution of this problem gives:

$$
\frac{m K_{p}}{b^{2}}=\frac{1}{1} \frac{\sqrt{1+\stackrel{2}{o}}}{2(1-\quad)}
$$

Under this choice, we have:

$$
G M_{1}=1 \sqrt{1+{ }_{o}^{2}} \text {. }
$$

Note that the right hand sides of (29) and (30) are functions of once 1 and $h=b / m$ are fixed.

Now, $\left(m K_{p} / b^{2}\right)$ is the controller gain, and to avoid actuator saturations this gain should not be too high. So, we can define a new cost function which tries to make $G M_{1}$ large and $K_{p}$ small:

$$
\operatorname{minimize}\left(\frac{2}{1 \sqrt{1+{ }_{o}^{2}}}+\frac{b^{2}}{m \quad 2} \frac{1}{1} \frac{\sqrt{1+{ }_{o}^{2}}}{2(1-\quad)}\right),
$$

where 2 assigns relative weights for $G M_{1}$ and $K_{p}$. Note that 1 does not play a role in the solution of (31). Once 2 and $h=b / m$ are fixed, the cost function defined in (31) depends $m$ on only. Minimizing the cost function gives optimal , then this gives $o$ and $K_{p}$ via (29); and once $K_{p}$ is known, we can find $K_{d}=m K_{p} / b$. Table 2 shows the optimal gains for varying 2 when $1=b^{2}=0.01, m=1$ and $h=b / m=0.005$ are fixed.

Table 2. Optimal gains and $G M_{1}$ for different 2, when $=0.05, m=1$, and $1=b^{2}=0.01$.

\begin{tabular}{||c||c|c|c|c|c|c|c|c||}
\hline 2 & 10 & 20 & 30 & 40 & 50 & 60 & 80 & 100 \\
\hline \hline$K_{p}$ & 94 & 207 & 301 & 389 & 425 & 436 & 446 & 453 \\
\hline$K_{d}$ & 2.4 & 6.3 & 12.7 & 34.3 & 82 & 127 & 207 & 291 \\
\hline$G M_{1}$ & 1.33 & 2.9 & 4.2 & 5.5 & 6.0 & 6.1 & 6.16 & 6.2 \\
\hline
\end{tabular}

Table 2 shows that $G M_{1}$ increases with increasing 2, but for $\quad 2 \geq 50$ additional gain in $G M_{1}$ is very small. Therefore, a meaningful choice would be $K_{p} \in[390,410]$ and $K_{d} \in$ $[35,45]$. Compared to the $H$ optimal gains corresponding to relatively large values, the above $K_{p}$ values are about 1.3 to 1.5 times higher, whereas $K_{d}$ values are 1.14 to 1.25 times lower. For the experimental tests, the values $K_{p}=400$ and $K_{d}=40$ are used and results are reported in the next section. These correspond to $2 \approx 42$ in the above table. For the $H$ optimal gains we may select $K_{p}=275$ and $K_{d}=45$; we expect the stability margins to be larger in this case, but the response will be slower. For relatively small values in the $H$ optimal design, we have $K_{p}=85$ and $K_{d}=15$ (e.g. for $b^{2}=1$ ) in which case the emphasis on tracking performance is diminished compared to larger values. In the next section experimental results for $K_{p}=400, K_{d}=40$ case and $K_{p}=85, K_{d}=15$ case will be illustrated.

\section{EXPERIMENTAL VALIDATION}

\subsection{Experimental setup}

In order to assure a full control of the communication delays and processing time, all the control algorithms (for haptic interface/virtual object) and virtual environment simulations will be run on the same computer.

The haptic interface, Figure $6 a$ and $b$, consists of three directdrive motor and three optical quadrature encoder with 1000 pts/rev (with a gear ratio of 1/10). The controllers and the virtual simulation are running in real time mode (on RTAI Linux) with a sampling time of $1 \mathrm{~ms}$. 

More precisely, for $K_{d}=40$, the maximum allowable $K_{p}$ is about 1000 . Considering the model uncertainties, the system's frictions and the operator's hand the system is still stable at this value. Another reason is that it is difficult to obtain high frequencies and the haptic interface input. Starting from $K_{p}=$ 1100 the system becomes unstable.

\section{CONCLUSIONS}

In this paper we have presented a complete stability analysis for a bilateral haptic system coupled to a virtual environment and affected by time delays. Using two optimization techniques we have proposed optimal controllers which were experimentally validated on 3 DOF haptic system in free and restricted motion.

To obtain good performance from the transparency point of view in free and restricted motion, using the same PD gains, we need to make a compromise in order to assure minimal performance in both cases. Another solution is to use a gain scheduling approach in order to switch from small to high gains depending on the case. A special attention it is needed for this approach because both controllers must be updated, and since the system is affected by time delays, there is a moment when the gains will be different at each side, moment that can induce unwanted effects and behaviors. The stability analysis in this case would fall into the framework of switched time delays systems and stability can be guaranteed for a sufficiently large dwell time, see for example Caliskan et al. (2011); Yan and Ozbay (2008); Yan et al. (2011) and their references.

A complete version of this article can be found in Liacu et al. (2012).

\section{ACKNOWLEDGEMENTS}

This work is supported in part by the French-Turkish PIA Bosphorus (TUBITAK Grant No. 109E127 and EGIDE Project No. 22974WJ). Hitay Özbay and Ahmet Taha Koru acknowledge partial financial support by DPT-HAMIT project. The work of Bogdan Liacu was financially supported by CEA LIST, Interactive Robotics Laboratory BP 6, 18 route du Panorama, F-92265 Fontenay-aux-Roses, France.

\section{REFERENCES}

Alfaro, V. (2007). PID controllers fragility. ISA Transactions, 46(4), 555-559.

Aziminejad, A., Tavakoli, M., Patel, R.V. and Moallem, M. (2008). Stability and performance in delayed bilateral teleoperation: Theory and experiments, Control Engineering Practice, 16, 1329-1343.

Çalışkan, S.Y., Özbay H., and Niculescu, S-I. (2011). Stability analysis of switched systems using Lyapunov-Krasovskii functionals. Preprints of the 18th IFAC World Congress, Milano (Italy) August 28 - September 2, 2011, pp. 74927496.

David, O., Measson, Y., Bidard, C., Rotinat-Libersa, C., and Russotto, F.X. (2007). Maestro: a hydraulic manipulator for maintenance and decomissionning. European Nuclear Conference (ENC), Bruxelles, Belgium.

Gil, J., Sanchez, E., Hulin, T., Preusche, C., and Hirzinger, G. (2007). Stability boundary for haptic rendering: Influence of damping and delay. In IEEE International Conference on Robotics and Automation,, $124-129$.
Gosselin, F., Mgard, C., Bouchigny, S., Ferlay, F., Taha, F., Delcampe, P., and D'Hauthuille, C. (2010). A VR training platform for maxillo facial surgery. Applied Human Factors and Ergonomics (AHFE) International Conference, Miami, Florida, USA, Advances in Cognitive Ergonomics.

Hokayem, P.F. and Spong, M.W. (2006). Bilateral teleoperation: An historical survey, Automatica, 42 (12) 2035-2057.

Keel, L. and Bhattacharyya, S. (1997). Robust, fragile or optimal? In Proceedings of the American Control Conference,, volume 2, $1307-1313$.

Lawrence, D.A. (1993). Stability and transparency in bilateral teleoperation. IEEE Trans. Robotics Automation, 9(5) 624637.

Lecuyer, A., Andriot, C., and Crosnier, A. (2003). Interfaces haptiques et pseudo-haptiques. Journees Nationales de la Recherche en Robotique.

Liacu B., Koru A.-T., Ozaby H., Niculescu S.-I., and Andriot C. (2012). Optimizing Low-Order Controllers for Haptic Systems under Delayed Feedback. Submitted to Control Engineering Practice.

Liacu B., Mendez-Barrios C., Niculescu S.-I., and Olaru S. (2010). Some remarks on the fragility of pd controllers for SISO systems with I/O delays. Proceedings 14th International Conference on System Theory and Control, Sinaia, Romania.

Makila, P., Keel, L., and Bhattacharyya, S. (1998). Comments on robust, fragile, or optimal? [and reply]. IEEE Transactions on Automatic Control, 43(9), 1265 -1268.

Michiels W., and Niculescu S.-I. (2007). Stability and stabilization of time-delay systems. An eigenvalue based approach. Advances in Design and Control 12, SIAM: Publications, Philadelphia.

Niemeyer, G. and Slotine, J.J.E. (2004). Telemanipulation with time delays. The International Journal of Robotics Research, 23(9), 873-890.

Nuno, E., Ortega, R., Barabanov, N., and Basanez, L. (2008). A globally stable pd controller for bilateral teleoperators. IEEE Transactions on Robotics, 24(3), $753-758$.

O'Dwyer, A. (2000). PI and PID controller tuning rules for time delay process: a summary. Technical report AOD-0001, Dublin Institute of Technology, Ireland.

Saeki, M. (2007). Properties of Stabilizing PID Gain Set in Parameter Space. IEEE Trans. Automat. Contr., vol. 52(9), 1710-1715.

Shayer, L.P., and Campbell, S.A. (2000). Stability, bifurcation and multistability in a system of two coupled neurons with multiple time delays. SIAM J. Appl. Math, 61(3), 673-700.

Sheridan, T.B. (1993). Space teleoperations through time delay: Review and prognosis. IEEE Trans. Robotics Automation 9, 592-606.

Silva, G.J., and Datta, A., and Bhattacharrya, S.P. (2005). PID Controllers for Time Delays Systems. Birkhauser, Boston.

Yan, P., and Özbay, H. (2008). Stability analysis of switched time-delay systems. SIAM J. Control and Optimization, vol. 47 (2008), no. 2, 936-949.

Yan, P., and Özbay, H., and Şansal, M. (2011). Dwell time optimization in switching control of parameter varying time delay systems. Proc. of the 50th IEEE Conference on Decision and Control, Orlando, USA, December 2011.

Yokokohji, Y. and Yoshikawa, T. (1994). Bilateral control of master-slave manipulators for ideal kinesthetic couplingformulation and experiment. IEEE Trans. Robotics Automation, 10(5), 605-619. 